\title{
Assessment of dynamic efforts taking into account of inertial and vibrating loads in deaxial pumping units
}

\author{
Beyali Ahmedov ${ }^{1}$ iD
}

Received: 28 August 2019 / Accepted: 8 January 2020 / Published online: 31 January 2020

(c) The Author(s) 2020

\begin{abstract}
The choice of rational designs of pumping units for lifting fluid from a well and the modes of their operation is one of the most urgent tasks in oil production. Despite the large amount of theoretical and extreme research, the solution of the problem in this direction cannot be considered complete, given that the dynamic characteristics of the movement of the well product and the kinematics of the well pumping unit are extremely complex. This article discusses the issue of determining the dynamic forces acting at the rod suspension point, in the new constructive solution of a beamless pumping unit depending on the angle of rotation of the crank and dimensionless kinematic parameter characterizing the relationship of the length of the crank to the length of the working part of the rope and the relative eccentric. Analytical dependencies are proposed to determine the maximum load at the rods suspension point depending on inertial and vibration forces.
\end{abstract}

Keywords Pumping unit · Balancer · Fluctuations · Rotor · Crank · Inertial load · Vibration load $\cdot$ Rod suspension point · Dynamic load

\section{List of symbols}

There are meaning of: $\mathrm{X}$ rated load on rod suspension point

XX maximum stroke length of rod

XXX

List of abbreviation

-СКД X-XX-XXX suspension point rated torque on the driven shaft of the gearbox

by OCT 26-16-08-87 (Industry standards) in Russian language means "станок-качалка дезаксиальный”-“deaxial pumping unit"

\section{Introduction}

In 2018, Azerbaijan produced 38 million and 758 thousand tons of crude oil (including gas condensate), which about $80 \%$ was operated by sucker-rod pumping unit. The most common drive sucker-rod pump is pumping unit. Every year

Beyali Ahmedov

ahmedov.beyali@mail.ru

1 Department of Machine Design, Azerbaijan Technical University, H. Javid Ave., 25, AZ 1073 Baku, Azerbaijan

their number is increasing due to the development of new territory, in particular, marine areas, and in the coming years this trend is expected to continue.

The pumping unit is a mechanism with one degree of freedom; therefore, the law of motion of the balancer and the associated rods column is determined by the geometric and kinematic parameters of the transforming mechanism. Depending on the location of the center of rotation of the cranks relative to the connecting point of rods with the balance bar, the mechanism of the pumping units can be axial or deaxial (offset), which is reflected in the performance of the pumping unit. At offset, or deaxial, crank mechanism the axis of the connecting-rod point with the balance bar does not intersect the axis of the crank shaft, but is displaced by a certain amount $(E)$. Such a mechanism is additionally characterized by the magnitude of the relative eccentricity $(\varepsilon)$. The main functional difference is that axial and deaxial types are based on the fact that axial transforming mechanisms reflect the characteristic meaning of continuous upward and downward moving forces are observed. Both schemes of mechanisms have their advantages and disadvantages; therefore, choosing one or the other type of mechanism, they strive to get the greatest benefits from the use of a rod installation in specific field conditions.

Along with axial crank mechanism (rocking machines type $\mathrm{CK}$ ), produced rocking machines with a deaxial scheme (type СКД). In the global industry (especially in the USA),

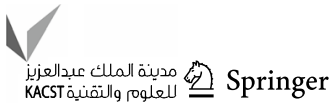


the vast majority of pumping units are produced with a slight deaxial. In recent years, deaxial rocking machines are increasingly being introduced, which in specific field conditions may be preferable to axial.

\section{Literature analysis and problem statement}

Rocking machines are an integral technique in the oil industry. At present, various pumping installations are used to operate oil wells in a mechanized way. The most common mechanized method of oil production is a suckerrod pumping machine with balanced individual drives, a machine of mechanical action-the so-called pumping units (CK) (Aliverdizade 1951; Chicherov et al. 1987). For many years, the studies have described the theory of work, analysis and synthesis of the kinematics of ordinary and unusual balancing individual drives, the law of change of efforts at the rod suspension point, the power calculation of pumping units and design features of typical types of balanced pumping units (Ivanovsky et al. 2002; Mishchenko 2003).

Various formulas have been utilized in determining the max load at the rod suspension point, depending on the pumping mode (Adonin 1973; Virnovsky 1971; Gimatudinov 1983). The most common in practice is the static method of calculating the maximum load at the rods suspension point, which are introduced at only the static force of gravity rods and fluid and the inertia force. The known calculation formulas for the maximum load in the upstroke have different spellings depending, mainly, on the method of estimating the inertial loads (the inertia of the fluid is taken into account or not), but the results of calculations on them are approximately the same.

For the kinematic study of the deaxial pumping unit (Abdullaev et al. 2016; Ahmedov et al. 2015; Ahmedov et al. 2018), the authors proposed new analytical expressions to determine the actual displacement, speed and acceleration of the suspension point of the rod. Several studies present the results of analytical studies and numerical calculations for a deaxial pumping unit.

So, as the elementary theory does not give the exact power calculation of pumping units, a satisfactory method of calculating the loads on the rods suspension point does not yet exist. The task of determining the efforts at the rods suspension point is very difficult. Attempts to solve it accurately lead to complex expressions, usually containing, difficult to define themselves.

The determination of the maximum inertial and vibration loads at the rods suspension point is a special interest.

\section{The purpose and objectives of the study}

The aim of the article is to determine the dynamic forces acting at the rods suspension point in a deaxial pumping unit, depending on the angle of rotation of the crank and dimensionless kinematic parameters, characterizing the ratios of the crank length to the connecting-rod length and relative eccentricity and obtaining an analytical expression for rate of dynamic forces.

To achieve this goal, it is necessary to solve the following tasks:

- Evaluation of the displacement, speed and acceleration of the rods suspension point when it is moving up and down in a deaxial pumping unit, depending on the angle of rotation of the crank and dimensionless kinematic parameters, characterizing the ratio of the length of the crank to the rod length and relative eccentricity;

- Obtaining analytical expressions for vibration and inertial forces;

- Obtaining an analytical expression for estimating the maximum efforts at the rod suspension point.

\section{Determination of the dynamic load acting on the rods during the operation of the well by the sucker-rod pumping unit}

One of the main parameters of the pumping unit is the load at the rods suspension point. This parameter largely determines the correct selection of the rocking machine, depending on the operating conditions of the pumping unit. There is a need to know the values of the factors that determine the amount of effort on the horse head (balancer head) and the method of their calculation. The main feature is that the loads acting at the rod suspension point vary in size, depending on the direction of the balancer head. In addition to static loads, due to the weight of the rod column, the fluid column inside the tubing string and the friction forces from the friction of the plunger on the cylinder and the rods on the pipes, dynamic loads act on the horse head (balancer head) - the forces of inertia of the movement of the column of rods, the fluid column and the vibration of the column of rods. The magnitude of these forces is determined by the ratio of the elements of the pumping unit and the number of double strokes. And the friction force is $2-5 \%$ of the magnitude of the static forces.

During the downhole pump operation cycle, loads on a column of pumping rods act as remain constant in size and direction throughout the entire cycle or a significant part of it, as well as variables. To constant or static loads, it is customary to attribute the weight of the rods column in a 
fluid $\left(G_{\text {rod }}\right)$, weight of a lifted fluid column $\left(G_{\text {fl }}\right)$, and the load from the friction of the rods on the walls of the lifting pipe $\left(G_{\text {fr.p }}\right)$ and from the friction of the plunger in the cylinder $\left(G_{\text {fr.c }}\right)$. And variable loads include $\left(G_{\text {in }}\right)$, due to the variable in magnitude and direction of the speed of movement of the system "rod-plunger," vibration load $\left(G_{\text {vib }}\right)$ due to oscillatory processes occurring in the rod column under the impact of shock application and removal of hydrostatic load on the plunger, load from friction of the rods in the fluid $\left(G_{\mathrm{fr} \cdot \mathrm{fl}}\right)$ and force of hydraulic resistance $\left(G_{h}\right)$ caused by the pressure drop in the discharge valve when the fluid moves.

Given the listed loads, we can write general formulas for determining the force at the rods suspension point when the rods move up and down:

$G_{u}=G_{\text {rod }}+G_{\text {fl }}+G_{\text {in } \cdot u}+G_{\text {vib } \cdot u}+G_{\text {fr } \cdot \mathrm{p}}+G_{\text {fr } \cdot \mathrm{c}}+G_{\text {fr.fl }}+G_{h}$

$G_{d}=G_{\mathrm{rod}}-\left(G_{\mathrm{in} \cdot \mathrm{d}}+G_{\mathrm{vib} \cdot \mathrm{d}}+G_{\mathrm{fr} \cdot \mathrm{p}}+G_{\mathrm{fr} \cdot \mathrm{c}}+G_{\mathrm{fr} \cdot \mathrm{fl}}+G_{h}\right)$

The calculation of the components of the friction force should be carried out for each specific case and is rather laborious. At the same time, not all components are significant in the general friction force, but require extensive information for the calculation, which is not always available.

Currently, there is no universal methodology for calculating extreme loads (maximum $G_{\max }$ and minimum $G_{\min }$ ), which would take into account all the enumerated force components acting on the rod string. However, extreme studies conducted in bench wells, and static processing of real field data made it possible to revealed that for a wide range of operating conditions of pumping plants and their operating modes in normal wells, the formulas of Virnovsky are most accurate (Virnovsky 1971):

$G_{u}=G_{\text {rod }}+G_{\text {fl }}+G_{\text {in } \cdot u}+G_{\text {vib.u }}$

$G_{d}=G_{\mathrm{rod}}-\left(G_{\mathrm{in} \cdot \mathrm{d}}+G_{\mathrm{vib} \cdot \mathrm{d}}\right)$

During the reciprocating movement of the rod, in addition to its own weight and the weight of the fluid column, which are static loads, the mechanical system is also loaded with dynamic forces that cause additional elastic deformations of the rods. The presence of dynamic loads arising from the uneven movement of the masses of the rods column, the uneven movement of the masses of the fluid column, as well as the rapid application and removal of the load from the weight of the fluid column when the plunger starts to move up and down, causes the rods of this mechanical system to vibrate.

Therefore, a comparative assessment of inertial and vibration loads caused by uneven movement of the masses of the rod column and the fluid column, taking into account the

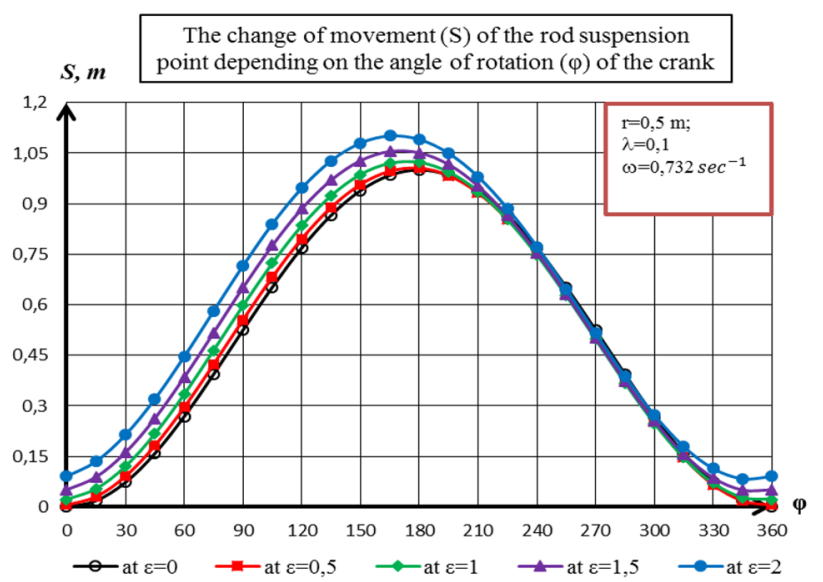

(a)

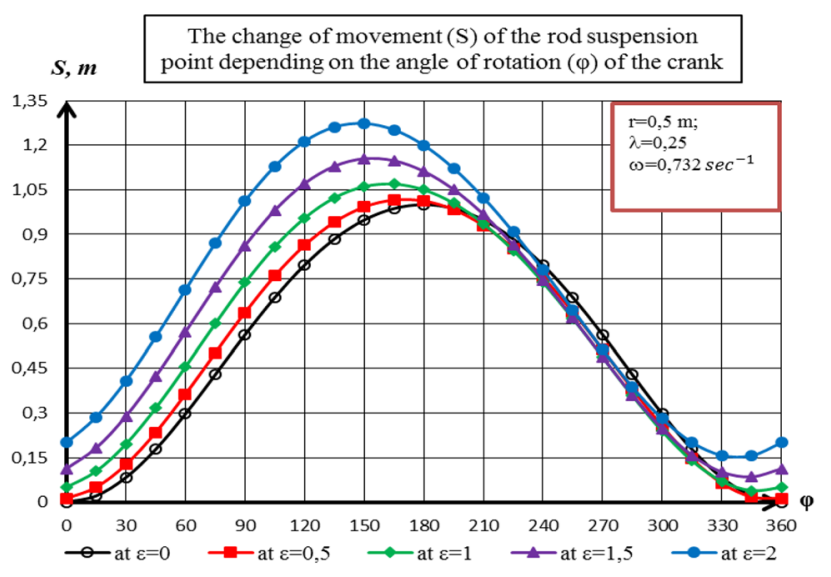

(b)

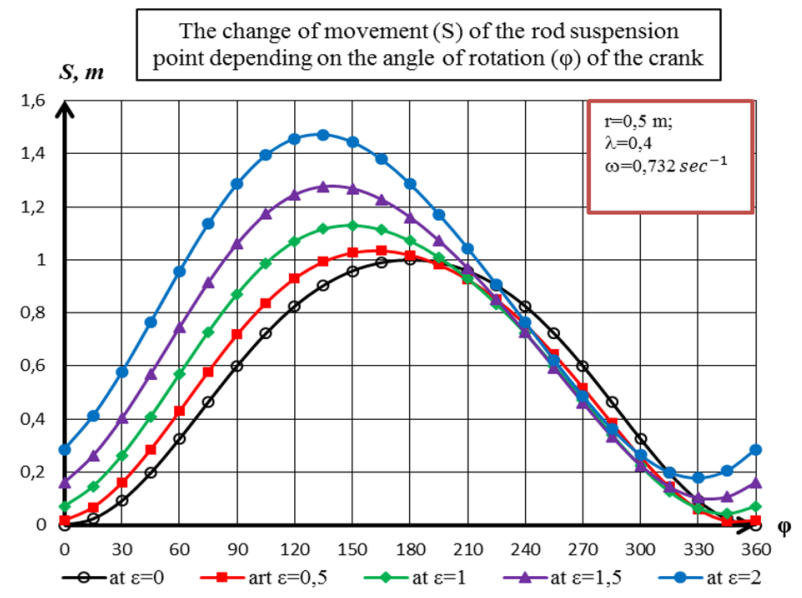

(c)

Fig. 1 The change of movement $(S)$ of the rod suspension point depending on the angle of rotation $(\varphi)$ of the crank

elastic deformations of the rods and pipes during one cycle of operation of the mechanical drive of the pumping, is of undoubted practical interest. 


\section{Determination of vibration forces in the pumping unit}

To determine the additional load at the rods suspension point from the vibration of the rods column during upstroke due to the sudden application of gravity of the fluid column above the plunger to their lower end for the pumping modes used, it will be determined by the formula (Aliverdizade 1951; Chicherov et al. 1987):

$G_{\text {vib }}=A_{\text {rod }} E \frac{v}{C}(1+0.3 k) \psi$

where $C$-the speed of sound in the metal; $v$-the speed of rods suspension point at the end of the deformation at the upstroke; $\psi$-coefficient depending on the ratio of the crosssectional areas of pump tubes and rods the relative eccentric $\varepsilon$ (Gimatudinov 1983; Abdullaev et al. 2016; Ahmedov et al. 2015).

For a deaxial pumping unit, the displacement of the rod suspension point is determined by the following expression:

$S=r\left[1-\cos \varphi+\frac{\lambda}{4}(1-\cos 2 \varphi)+\varepsilon \lambda \sin \varphi-\frac{\varepsilon^{2} \lambda^{2}}{2(1+\lambda)}+\frac{\lambda \varepsilon^{2}}{2}\right]$

where $r$ - crank radius; $\omega$-the angular velocity of the crank; $\varphi$-crank angle rotation; $\lambda=\frac{r}{l}$-dimensionless kinematic parameter characterizing the relationship of the crank length to the length of the working part of the rope of the pumping unit; $\varepsilon=\frac{E}{r}$ relative eccentricity; $E$-deaxial value.

In pumping units with the same $r$ and $\lambda$, the movement of the rods suspension point in the deaxial mechanisms is slightly larger than in the case of axial ones.

To determine the speed of the rods suspension point, we differentiate expression (6) over time

$$
\begin{aligned}
V=\frac{\mathrm{d} S}{\mathrm{~d} t} & =\frac{d\left[r\left(1-\cos \varphi+\frac{\lambda}{4}(1-\cos 2 \varphi)+\varepsilon \lambda \sin \varphi-\frac{\varepsilon^{2} \lambda^{2}}{2(1+\lambda)}+\frac{\lambda \varepsilon^{2}}{2}\right)\right]}{\mathrm{d} t} \\
& =r\left[0-\left(-\sin \frac{\mathrm{d} \varphi}{\mathrm{d} t}\right)+\frac{\lambda}{4}\left[0-\left(-\sin 2 \varphi \cdot 2 \frac{\mathrm{d} \varphi}{\mathrm{d} t}\right)\right]+\varepsilon \lambda \cos \varphi \frac{\mathrm{d} \varphi}{\mathrm{d} t}-0+0\right] \\
& =r \cdot \omega\left[\sin \varphi+\frac{\lambda}{2} \sin 2 \varphi+\varepsilon \lambda \cos \varphi\right]
\end{aligned}
$$

$\psi=\frac{A_{\mathrm{p}}}{A_{\mathrm{p}}+A_{\mathrm{rod}}}=\frac{\psi_{P}^{2}}{\psi_{p}^{2}+1}$

$k=\left(\frac{A_{\mathrm{pl}}-A_{\mathrm{rod}}}{A_{\mathrm{pl}}-A_{\mathrm{rod}}}\right) \frac{G_{\mathrm{fl}}}{G_{\mathrm{rod}}}=\left(\frac{\psi_{\mathrm{pl}}^{2}-1}{\psi_{\mathrm{pl}}^{2}-1}\right) \frac{G_{\mathrm{fl}}}{G_{\mathrm{rod}}}$

where $G_{\mathrm{rod}}$-the force of gravity in the air bars; $G_{\mathrm{ff}}$-the force of gravity of the fluid displaced by rods; $A_{\mathrm{pl}}$-sectional area of the plunger; $A_{\mathrm{p}}$ - sectional area of the pumping pipe; $A_{\text {rod }}$-sectional area of the rod;

$\psi_{\mathrm{pl}}=\frac{D_{\mathrm{pl}}}{D_{\mathrm{rod}}} ; \psi_{p}=\frac{D_{\mathrm{p}}}{D_{\mathrm{rod}}}$ dimensionless parameters which characterized the ratio of the diameter of the plunger and the pipe to the diameter of the rod.

The intensity of oscillations caused by the speed of the pump valves largely depends on the speed of the rod suspension point. Practice shows that sometimes it is sufficient to increase or decrease the number of swings of the rod suspension point by only one per minute so that the load on the rod decreases.

In many tasks of the dynamics of transforming mechanisms of the pumping unit, it is more convenient to use relations in which the displacement of the rod suspension point $S$ is a function of the angle of rotation of the $\operatorname{crank} \varphi$ depending on the dimensionless kinematic parameter $\lambda$ and
Based on the calculation results, taking into account Eqs. (6) and (7) are constructed graphs of displacement $(S)$ (Fig. 1) and the (v) velocity (Fig. 2) the rods suspension point on the angle of rotation of the crank $(\varphi)$ of the pumping unit at $r=0.5 \mathrm{~m}, \omega=0.732 \mathrm{sec}^{-1}$ for different values of the dimensionless kinematic parameter $\lambda$ and the relative eccentric $\varepsilon$.

As can be seen from these figures with a relative eccentricity $\varepsilon=0$, we have a special case for the axial mechanism. In this case, the maximum value of the displacement of the rods suspension point is practically equal to $S=2 r$ (Fig. 1a), which corresponds to the angle of rotation of the crank at $\varphi=180^{\circ}$. In this case, when the rods suspension point is moved up and down the angles of rotation of the crank equally $\varphi_{1}=\varphi_{1}^{\prime}$, and an extreme value of the speed of rods suspension point, respectively, obtained by rotating crank $\varphi=90^{\circ} ; \varphi=270^{\circ}$ (Fig. 1a). However, as the relative eccentricity $(\varepsilon)$ and dimensionless kinematic parameter $(\lambda)$ crank rotation angles differ $\varphi_{1} \neq \varphi_{1}^{\prime}$ increases and the speed of movement of the rod suspension point to vary crank angle corresponding to these values (deaxial mechanism). As can be seen from the graphs with $\lambda=0.4$ and $\varepsilon=2$, the maximum value of the displacement of the rods suspension point in this case increases almost to $S \approx 3 r$ (Fig. 1c). 


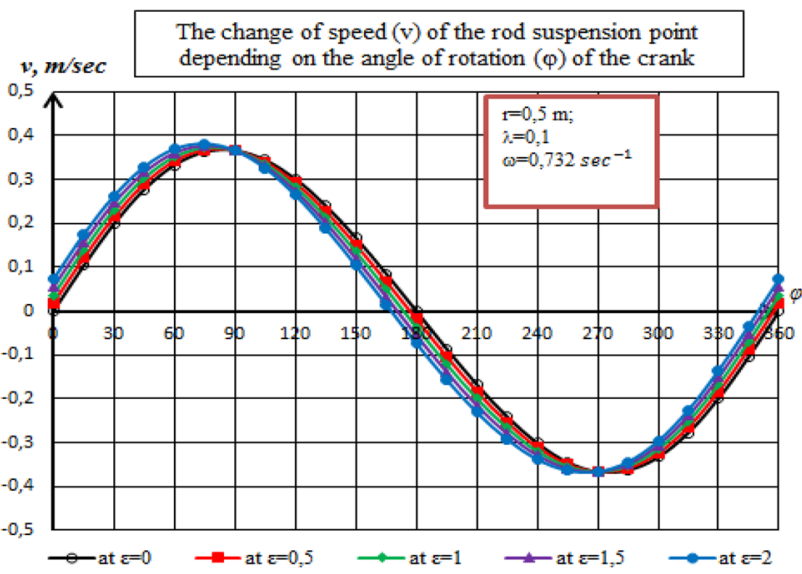

(a)

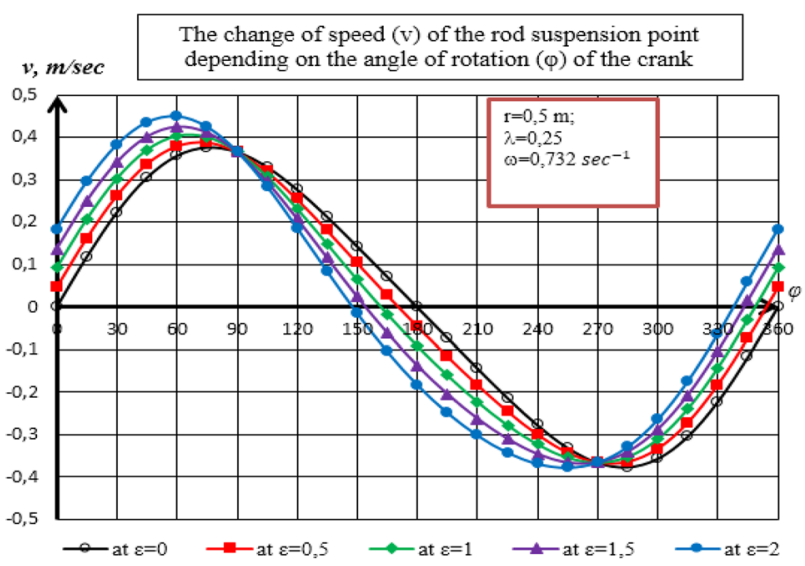

(b)

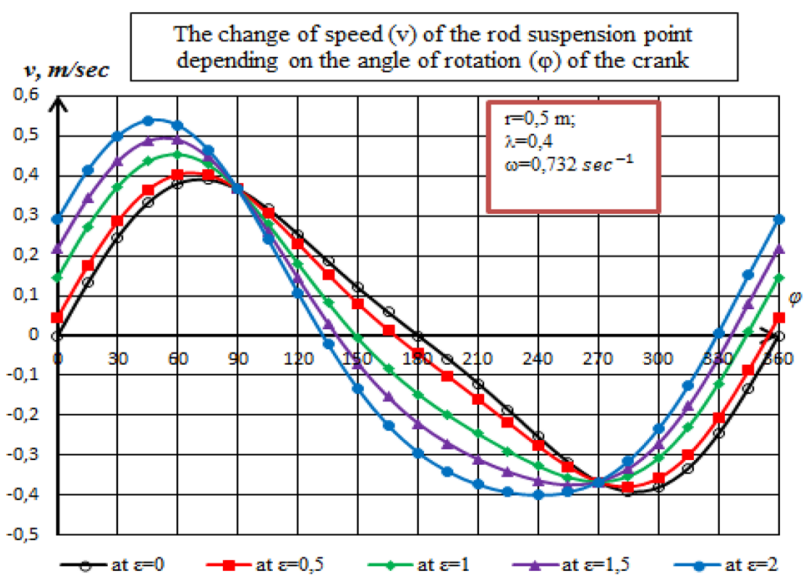

(c)

Fig. 2 The change of speed ( $v$ ) of the rod suspension point depending on the angle of rotation $(\varphi)$ of the crank

This is explained by the fact that the greatest displacement of the rods suspension point is achieved not at $\varphi=180^{\circ}$, but at $\varphi=132^{\circ}$ (Fig. 1c). In this case, the speed of the rods suspension point will receive extreme values, respectively, at the angles of rotation of the crank to $\varphi=49^{\circ} 33^{\prime}$ and $\varphi=240^{\circ} 42^{\prime}$ (Fig. 2c)

For the analytical determination of the angles of rotation of the crank at which the speed of the rods suspension point turns out to be extreme, we find by equating to zero the derivative of the right side of Eq. (7)

$\frac{\mathrm{d} V}{\mathrm{~d} \varphi}=r \cdot \omega(\cos \varphi+\lambda \cos 2 \varphi-\varepsilon \lambda \sin \varphi)$

$\cos \varphi+\lambda \cos 2 \varphi-\varepsilon \lambda \sin \varphi=0$

From where

$2 \lambda \sin ^{2} \varphi+\varepsilon \lambda \sin \varphi-\cos \varphi-\lambda=0$

Expressing the trigonometric function sine through the tangent of the half argument, we get

$\sin \varphi=\frac{2 \operatorname{tg} \frac{\varphi}{2}}{1+\operatorname{tg}^{2} \frac{\varphi}{2}} ; \quad \cos \varphi=\frac{1-\operatorname{tg}^{2} \frac{\varphi}{2}}{1+\operatorname{tg}^{2} \frac{\varphi}{2}} ;$

by replacing $\operatorname{tg} \frac{\varphi}{2}=x$, we get

$2 \lambda\left(\frac{2 x}{1+x^{2}}\right)^{2}+\varepsilon \lambda \frac{2 x}{1+x^{2}}-\frac{1-x^{2}}{1+x^{2}}-\lambda=0$

$(1-\lambda) x^{4}+2 \varepsilon \lambda x^{3}+6 \lambda x^{2}+2 \varepsilon \lambda x-(1+\lambda)=0$

If we replace $(1-\lambda)=A, \quad 2 \varepsilon \lambda=B, \quad 6 \lambda=C$ and $-(1+\lambda)=D$, we obtain the following equation of the fourth degree

$A x^{4}+B x^{3}+C x^{2}+B x+D=0$

There are various ways to find the roots of this equation, respectively, the values of the angles of rotation of the crank $\varphi$ at which the piston speed $v$ turns out to be extreme (for example, the method of auxiliary coefficients, Ferrari, Descartes-Euler, Laguerre or using an online calculator, introducing the coefficients $A, B, C, D$ ).

The speed of the rods suspension point and the angles of rotation of the crank corresponding to this value for different values of $\lambda$ and $\varepsilon$ are given in Table 1 .

According to the found value of the speed of the rods suspension point, we get the vibration force during upstroke:

$G_{\text {vib }}=A_{\text {rod }} \frac{E}{C} r \cdot \omega\left[\sin \varphi+\frac{\lambda}{2} \sin 2 \varphi+\varepsilon \lambda \cos \varphi\right]\left[1+0.3 k^{\prime} \frac{G_{\text {fl }}}{G_{\text {rod }}}\right] \psi$

where $k^{\prime}=\left(\frac{\psi_{\mathrm{p}}^{2}-1}{\psi_{\mathrm{p}}^{2}-1}\right)$

Based on the calculations performed for a deaxial pumping unit, it was found that for different values of relative eccentricity $(\varepsilon=0 ; \varepsilon=0.5 ; \varepsilon=1.0 ; \varepsilon=1.5$ and $\varepsilon=2.0)$ and dimensionless kinematic parameter $(\lambda=0.1, \lambda=0.25$ and 
Table 1 The value of the maximum speed of the rods suspension point at the corresponding angles of rotation of the crank, depending on the dimensionless parameters $\lambda$ and $\varepsilon$

\begin{tabular}{llll}
\hline$\lambda$ & $\varepsilon$ & $\varphi$ & $v_{\max }(\mathrm{m} / \mathrm{s})$ \\
\hline 0.1 & 0.5 & $\varphi_{1 \max }=81^{\circ} 38^{\prime} ; \varphi_{1 \max }=272^{\circ} 52^{\prime}$ & $v_{\max }=0.3700 ; v_{\max }=-0.3665$ \\
& 1.0 & $\varphi_{1 \max }=79^{\circ} 01^{\prime} ; \varphi_{1 \max }=270^{\circ} 02^{\prime}$ & $v_{\max }=0.3731 ; v_{\max }=-0.3660$ \\
& 1.5 & $\varphi_{1 \max }=76^{\circ} 24^{\prime} ; \varphi_{1 \max }=267^{\circ} 36^{\prime}$ & $v_{\max }=0.3770 ; v_{\max }=-0.3665$ \\
& 2.0 & $\varphi_{1 \max }=73^{\circ} 54^{\prime} ; \varphi_{1 \max }=264^{\circ} 14^{\prime}$ & $v_{\max }=0.3817 ; v_{\max }=-0.3678$ \\
& 0.5 & $\varphi_{1 \max }=71^{\circ} 28^{\prime} ; \varphi_{1 \max }=276^{\circ} 50^{\prime}$ & $v_{\max }=0.3891 ; v_{\max }=-0.3687$ \\
& 1.0 & $\varphi_{1 \max }=66^{\circ} 28^{\prime} ; \varphi_{1 \max }=270^{\circ} 00^{\prime}$ & $v_{\max }=0.4056 ; v_{\max }=-0.3660$ \\
& 1.5 & $\varphi_{1 \max }=61^{\circ} 56^{\prime} ; \varphi_{1 \max }=262^{\circ} 32^{\prime}$ & $v_{\max }=0.4255 ; v_{\max }=-0.3689$ \\
& 2.0 & $\varphi_{1 \max }=57^{\circ} 52^{\prime} ; \varphi_{1 \max }=254^{\circ} 28^{\prime}$ & $v_{\max }=0.4485 ; v_{\max }=-0.3781$ \\
& 0.5 & $\varphi_{1 \max }=64^{\circ} 28^{\prime} ; \varphi_{1 \max }=280^{\circ} 14^{\prime}$ & $v_{\max }=0.4187 ; v_{\max }=-0.3728$ \\
& 1.0 & $\varphi_{1 \max }=58^{\circ} 30^{\prime} ; \varphi_{1 \max }=270^{\circ} 02^{\prime}$ & $v_{\max }=0.4538 ; v_{\max }=-0.3660$ \\
& 1.5 & $\varphi_{1 \max }=53^{\circ} 22^{\prime} ; \varphi_{1 \max }=257^{\circ} 00^{\prime}$ & $v_{\max }=0.4948 ; v_{\max }=-0.3739$ \\
& 2.0 & $\varphi_{1 \max }=49^{\circ} 33^{\prime} ; \varphi_{1 \max }=240^{\circ} 42^{\prime}$ & $v_{\max }=0.5408 ; v_{\max }=-0.3999$ \\
\hline
\end{tabular}

$\lambda=0.4)$, the maximum movement of the rods suspension point is $S=(2.0 \ldots 3.0) r$, and its maximum speed at the largest value of dimensionless kinematic parameter (in pumping units brand СКД2-0,6-250, СКД4-2,1-1400, СКД6-2,52800, СКД8-3-4000, СКД10-3,5-5600 and СКД12-3-5600 the value of dimensionless kinematic parameter is $\lambda=0.4$ ) varies within $v_{\max }=(1.05 \ldots 1.48) r \omega^{2}$. Therefore, with average values of the displacement of the rods suspension point $S \approx 2.5 r$ and speed $v_{\max } \approx 1.27 r \omega$, the expression of the vibration force with regard to formula (12) takes the following form:

$G_{\mathrm{vib}}=\left[1+0.3 k^{\prime} \frac{G_{\mathrm{fl}}}{G_{\mathrm{rod}}}\right] \psi \frac{E}{C} \frac{\pi D_{\mathrm{rod}}^{2}}{4} 1.27 \cdot \frac{S}{2.5} \cdot \frac{\pi n}{30}$

If given that the values of $\frac{\pi}{4} 1.27 \cdot \frac{S}{2.5} \frac{\pi n}{30}=\frac{3.14^{2} \cdot 1.27}{4 \cdot 2.5 \cdot 30}$ $S n=\frac{S n}{24 \cdot 60}=\frac{S n}{1440}$ then the expression of the dynamic force in upstroke will take the form

$G_{\mathrm{vib}}=\frac{S n}{1440}\left[1+0.3 k^{\prime} \frac{G_{\mathrm{fl}}}{G_{\mathrm{rod}}}\right] \psi \frac{E}{C} D_{\mathrm{rod}}^{2}$

\section{Determination of inertia forces in the pumping unit}

Consider the change in the inertial load acting on the polished rod during the operation of the well sucker-rod pumping unit. The value of inertial loads in the column of rods depends on the type of pumping unit and on the direction of rotation of the crank.

We denote the inertial force in the rods suspension point on the upstroke $-G_{\text {in.u }}$, on the downstroke $-G_{\text {in·d }}$ and define the dynamic forces
$G_{\mathrm{in} \cdot \mathrm{u}}=m a=\frac{G_{\mathrm{rod}}+G_{\mathrm{fl}}}{g} a=\frac{a}{g}\left(G_{\mathrm{rod}}+G_{\mathrm{fl}}\right)$

$G_{\text {in } \cdot \mathrm{d}}=m^{\prime} a=\frac{G_{\mathrm{rod}}}{g} a=\frac{a}{g} G_{\mathrm{rod}}$

where $a$-acceleration of the rods suspension point; $g$ acceleration of gravity.

To find acceleration of the rods suspension point for deaxial pumping unit, we differentiate expression (7) by time

$$
\begin{aligned}
a=\frac{\mathrm{d} V}{\mathrm{~d} t} & =\frac{d\left[r \cdot \omega\left(\sin \varphi+\frac{\lambda}{2} \sin 2 \varphi+\varepsilon \lambda \cos \varphi\right)\right]}{\mathrm{d} t} \\
& =r \cdot \omega\left[\cos \varphi \frac{\mathrm{d} \varphi}{\mathrm{d} t}+\frac{\lambda}{2} \cos 2 \varphi \cdot 2 \frac{\mathrm{d} \varphi}{\mathrm{d} t}+\varepsilon \lambda\left(-\sin \varphi \frac{\mathrm{d} \varphi}{\mathrm{d} t}\right)\right] \\
& =r \cdot \omega^{2}[\cos \varphi+\lambda \cos 2 \varphi-\varepsilon \lambda \sin \varphi]
\end{aligned}
$$

Consider how the acceleration of the rods suspension point varies with up- and downstroke depending on the angle of rotation of the crank $\varphi$.

Figure 3 shows a graph of the acceleration of the rods suspension point $(a)$ on the angle of rotation $(\varphi)$ of the crank. As can be seen from Fig. 3a, with relative eccentricity $\varepsilon=0$ (axial mechanism), the extreme value of acceleration is obtained by turning the crank by $\varphi=0^{\circ}$ and $\varphi=180^{\circ}$. However, increasing both the relative eccentricity and kinematic dimensionless parameter increases acceleration of the rods suspension point and crank angle is changed corresponding to the value (deaxial mechanism). As shown in the graphs, at $\lambda=0.25$ and $\varepsilon=2$, the extreme values of acceleration are obtained not at $\varphi=0^{\circ}$ and $\varphi=180^{\circ}$, but by turning the crank to $\varphi=127^{\circ} 46^{\prime}$ and $\varphi=345^{\circ} 46$ ' (Fig. 3b). When $\lambda=0.4$ and $\varepsilon=2$, the acceleration of the rods suspension point increases sharply and the angle of rotation of the crank corresponding to this value changes. As can be seen from the 


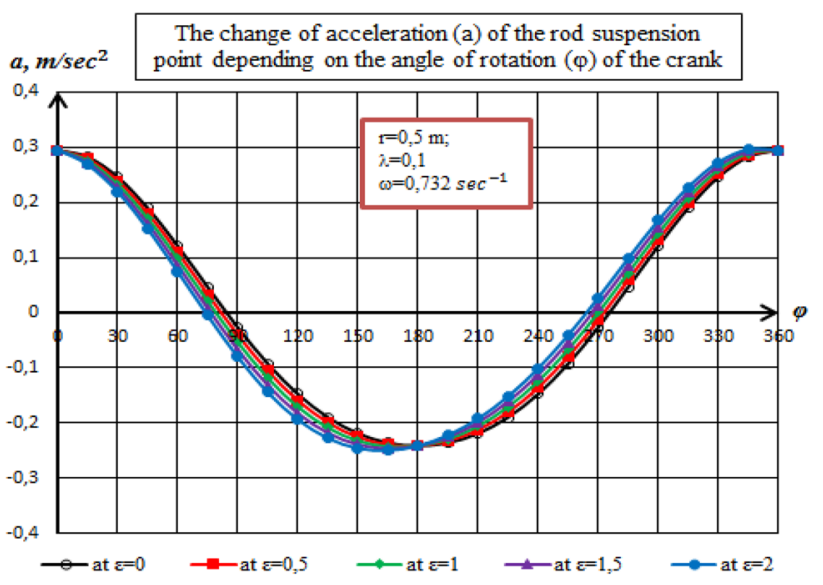

(a)

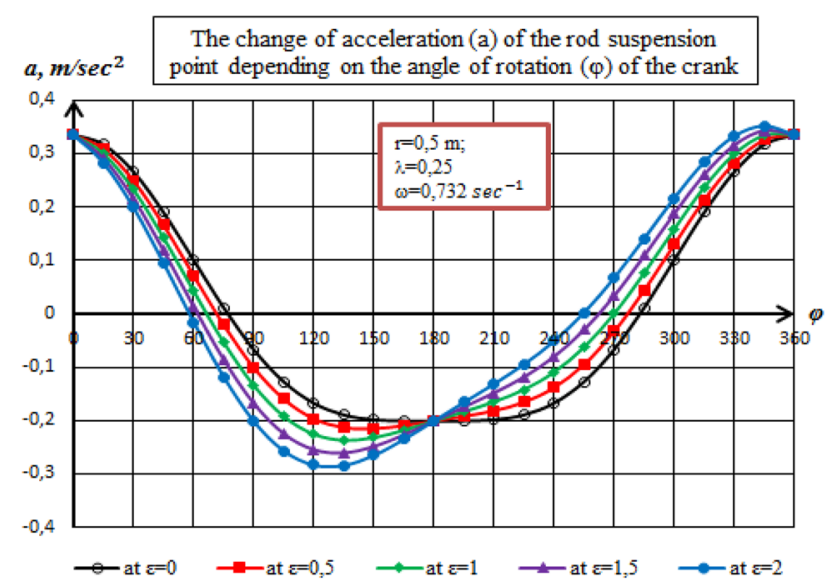

(b)

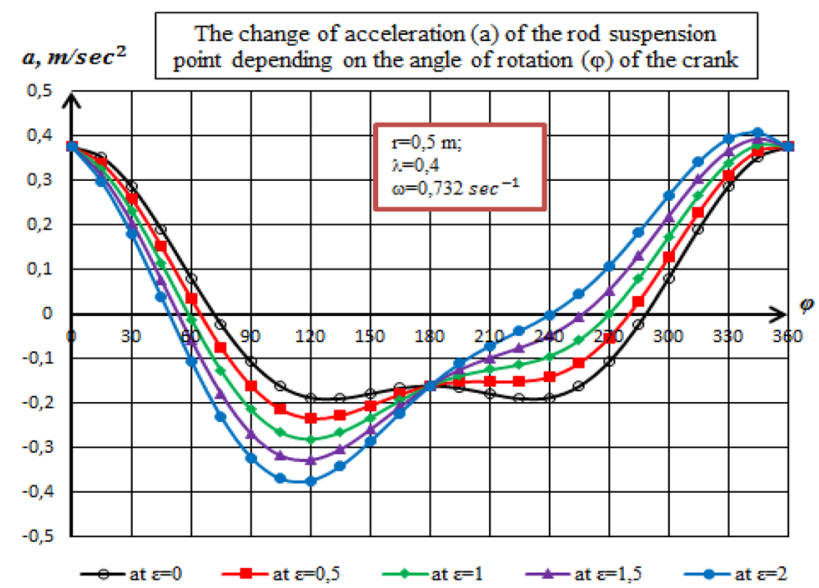

(c)

Fig. 3 The graphs of the acceleration of the rod suspension point $\left(a_{E}\right)$ on the angle of rotation of the crank $(\varphi)$

graphs, the extreme value of the acceleration of the rods suspension point in this case is obtained when the angle of rotation of the crank at $\varphi=113^{\circ} 50^{\prime}$ and $\varphi=342^{\circ} 25^{\prime}$ (Fig. 3c).

For the analytical determination of the values of rotation angles of the crank $\varphi$ at which the acceleration of the piston $a$ turns out to be extreme, we find that the derivative with respect to $\varphi$ from the right side of the equation is equal to zero

$\frac{\mathrm{d} a}{\mathrm{~d} \varphi}=r \omega^{2}(-\sin \varphi-2 \lambda \sin 2 \varphi-\varepsilon \lambda \cos \varphi)$

From where

$\sin \varphi+4 \lambda \sin \varphi \cos \varphi+\varepsilon \lambda \cos \varphi=0$

Expressing the trigonometric function sine through the tangent of the half argument, we get

$\sin \varphi=\frac{2 \operatorname{tg} \frac{\varphi}{2}}{1+\operatorname{tg}^{2} \frac{\varphi}{2}} ; \quad \cos \varphi=\frac{1-\operatorname{tg}^{2} \frac{\varphi}{2}}{1+\operatorname{tg}^{2} \frac{\varphi}{2}} ;$

by replacing $\operatorname{tg} \frac{\varphi}{2}=x$, we get

$$
\begin{array}{r}
\frac{2 x}{1+x^{2}}+4 \lambda\left(\frac{2 x}{1+x^{2}}\right)\left(\frac{1-x^{2}}{1+x^{2}}\right)+\varepsilon \lambda\left(\frac{1-x^{2}}{1+x^{2}}\right)=0 \\
-\varepsilon \lambda x^{4}+2 x^{3}(1-4 \lambda)+2 x(1+4 \lambda)+\varepsilon \lambda=0
\end{array}
$$

If we replace $\varepsilon \lambda=A ; 2(1-4 \lambda)=B$ and $2(1+4 \lambda)=C$ we get the following equation of the fourth degree

$A x^{4}-B x^{3}-C x-A=0$

The values of the acceleration of the rods suspension point and the angles of rotation of the crank corresponding to this value for different values of $\lambda$ and $\varepsilon$ are given in Table 2.

According to the found value of mass and acceleration, we obtain a dynamic force during the upstroke

$G_{\text {in }}=\frac{G_{\text {rod }}+G_{\text {fl }}}{g} r \omega^{2}(\cos \varphi+\lambda \cos 2 \varphi-\varepsilon \lambda \sin \varphi)$

Dynamic force on the downstroke

$G_{\text {in }}^{\prime}=\frac{G_{\text {rod }}}{g} r \omega^{2}(\cos \varphi+\lambda \cos 2 \varphi-\varepsilon \lambda \sin \varphi)$.

Based on the calculations performed for a deaxial rocking machine, it was found that for different values of relative eccentricity and dimensionless kinematic parameter, the maximum displacement of the rods suspension point is $S_{\max }=(2.0 \ldots 3.0) r$, and its maximum acceleration at the highest value of the dimensionless kinematic parameter varies within $a_{\max }=(1.41 \ldots 1.52) r \omega^{2}$. Therefore, with average values of displacement $S \approx 2.5 r$ and acceleration $a_{\max } \approx 1.47 r \omega^{2}$, the expression of inertial force takes the following form: on the upstroke

$G_{\text {in }}=\frac{S \cdot \pi^{2} \cdot n^{2}}{2.5 \cdot 30^{2} g} 1.47\left(G_{\text {rod }}+G_{\text {fl }}\right)$

on the downstroke 
Table 2 The value of the maximum acceleration of the rods suspension point at the corresponding angles of rotation of the crank, depending on the dimensionless parameters $\lambda$ and $\varepsilon$

\begin{tabular}{llll}
\hline$\lambda$ & $\varepsilon$ & $\varphi$ & $a_{E \max }\left(\mathrm{m} / \mathrm{s}^{2}\right)$ \\
\hline 0.1 & 0.5 & $\varphi_{1 \max }=175^{\circ} 14^{\prime} ; \varphi_{1 \max }=357^{\circ} 58^{\prime}$ & $a_{\max }=-0.2417 ; a_{\max }=0.2949$ \\
& 1.0 & $\varphi_{1 \max }=170^{\circ} 37^{\prime} ; \varphi_{1 \max }=355^{\circ} 56^{\prime}$ & $a_{\max }=-0.2433 ; a_{\max }=0.2956$ \\
& 1.5 & $\varphi_{1 \max }=166^{\circ} 13^{\prime} ; \varphi_{1 \max }=353^{\circ} 54^{\prime}$ & $a_{\max }=-0.2460 ; a_{\max }=0.2968$ \\
& 2.0 & $\varphi_{1 \max }=162^{\circ} 06^{\prime} ; \varphi_{1 \max }=351^{\circ} 52^{\prime}$ & $a_{\max }=-0.2497 ; a_{\max }=0.2985$ \\
0.25 & 0.5 & $\varphi_{1 \max }=145^{\circ} 08^{\prime} ; \varphi_{1 \max }=356^{\circ} 26^{\prime}$ & $a_{\max }=-0.2158 ; a_{\max }=0.3359$ \\
& 1.0 & $\varphi_{1 \max }=137^{\circ} 12^{\prime} ; \varphi_{1 \max }=352^{\circ} 52^{\prime}$ & $a_{\max }=-0.2369 ; a_{\max }=0.3391$ \\
& 1.5 & $\varphi_{1 \max }=131^{\circ} 44^{\prime} ; \varphi_{1 \max }=349^{\circ} 18^{\prime}$ & $a_{\max }=-0.2609 ; a_{\max }=0.3442$ \\
& 2.0 & $\varphi_{1 \max }=127^{\circ} 46^{\prime} ; \varphi_{1 \max }=345^{\circ} 46^{\prime}$ & $a_{\max }=-0.2867 ; a_{\max }=0.3515$ \\
& 0.5 & $\varphi_{1 \max }=122^{\circ} 56^{\prime} ; \varphi_{1 \max }=355^{\circ} 36^{\prime}$ & $a_{\max }=-0.2344 ; a_{\max }=0.3771$ \\
& 1.0 & $\varphi_{1 \max }=119^{\circ} 04^{\prime} ; \varphi_{1 \max }=351^{\circ} 12^{\prime}$ & $a_{\max }=-0.2804 ; a_{\max }=0.3833$ \\
& 1.5 & $\varphi_{1 \max }=116^{\circ} 08^{\prime} ; \varphi_{1 \max }=346^{\circ} 49^{\prime}$ & $a_{\max }=-0.3279 ; a_{\max }=0.3935$ \\
& 2.0 & $\varphi_{1 \max }=113^{\circ} 50^{\prime} ; \varphi_{1 \max }=342^{\circ} 25^{\prime}$ & $a_{\max }=-0.3765 ; a_{\max }=0.4077$ \\
\hline
\end{tabular}

$G_{\text {in }}^{\prime}=\frac{S \cdot \pi^{2} \cdot n^{2}}{2.5 \cdot 30^{2} g} 1.47 G_{\mathrm{rod}}$

where $S$ - the movement of the rods suspension point; $n-$ number of double moves of pumping unit.

If given that the values of $\pi^{2}=9.87$ and $g=9.81$ are approximately equal, they can be reduced. Because of $\frac{2.5 \cdot 30^{2}}{1.47}=1530$, the expression of dynamic force at the upstroke takes the form

$G_{\text {in }}=\frac{S n^{2}}{1530}\left(G_{\text {rod }}+G_{\text {fl }}\right)$

And on the downstroke

$G_{\text {in }}=\frac{S n^{2}}{1530} G_{\text {rod }}$

where $\frac{a}{g}=\psi_{d}=\frac{S n^{2}}{1530}$-dynamic factor.

From (25) and (26), it follows that the dynamic factor and inertial load increase in proportion to the length of the stroke and the square of the number of double strokes of the pumping unit.

Then considering the listed load, we can record efforts at the rods suspension point on the upstroke

$G_{\max }=\left(G_{\text {rod }}+G_{\text {fl }}\right)\left(1+\frac{S n^{2}}{1530}\right)+\frac{S n}{1440}\left(1+0.3 k^{\prime} \frac{G_{\mathrm{fl}}}{G_{\text {rod }}}\right) \psi \frac{E}{C} D_{\text {rod }}^{2}$

And on the downstroke

$G_{\text {min }}=G_{\text {rod }}\left(1-\frac{S n^{2}}{1530}\right)-\frac{S n}{1440}\left(1+0.3 k^{\prime} \frac{G_{\text {fl }}}{G_{\text {rod }}}\right) \psi \frac{E}{C} D_{\text {rod }}^{2}$

\section{Results}

1. Based on the obtained analytical dependencies, it has been proved that changes in the magnitude of dynamic loads per cycle at given geometrical values of the pumping unit and its mode of operation depend not only on the angle of rotation of the $\operatorname{crank} \varphi$, but also on the dimensionless kinematic parameter $\lambda$ and relative eccentricity $\varepsilon$.

2. It is found that the maximum value of the dynamic load when $\varepsilon=0$ and $\lambda=0.1$ is achieved with angles of rotation of the $\operatorname{crank} \varphi=0^{\circ}, 180^{\circ}, 360^{\circ}$. And with an increase in the relative eccentricity, the angles of rotation of the crank corresponding to the maximum value of the dynamic forces are $\varphi_{\max }=162^{\circ}-175^{\circ}$ and $\varphi_{\max }=351^{\circ}-357^{\circ}$.

3. With an increase in the dimensionless kinematic parameter $\lambda$, the maximum value of dynamic loads increases dramatically. Because of, at $\lambda=0.25$, the angles of rotation of the crank corresponding to the maximum value of the dynamic forces are $\varphi_{\max }=127^{\circ}-145^{\circ}$ and $\varphi_{\max }=345^{\circ}-356^{\circ}$, and at $\lambda=0.4$ it is $\varphi_{\max }=113^{\circ}-122^{\circ}$ and $\varphi_{\max }=342^{\circ}-355^{\circ}$.

4. The proposed analytical expressions allow to determine the value of the maximum dynamic force at the rods suspension point, depending on the value of static loads.

5. Based on the proposed methodology, determined the real maximum load acting at the rods suspension point of a deaxial pumping unit, taking into account inertial and vibration loads.

6. The results of the calculations allow to be guided in determining the maximum load acting at the rods suspension point and the choice of the appropriate size of the pumping unit. 
Acknowledgements Not applicable.

Funding Not applicable.

Availability of data and materials The datasets supporting the conclusions of this article are included within the article.

\section{Compliance with ethical standards}

Conflict of interests The authors declare no competing financial interests.

Consent for publication Not applicable.

Ethics approval and consent to participate Not applicable.

Open Access This article is licensed under a Creative Commons Attribution 4.0 International License, which permits use, sharing, adaptation, distribution and reproduction in any medium or format, as long as you give appropriate credit to the original author(s) and the source, provide a link to the Creative Commons licence, and indicate if changes were made. The images or other third party material in this article are included in the article's Creative Commons licence, unless indicated otherwise in a credit line to the material. If material is not included in the article's Creative Commons licence and your intended use is not permitted by statutory regulation or exceeds the permitted use, you will need to obtain permission directly from the copyright holder. To view a copy of this licence, visit http://creativecommons.org/licenses/by/4.0/.

\section{References}

Abdullaev AH, Najafov AM, Ahmedov BB (2016) New mechanical drive for sucker rod pumps for oil production, Scientific, technical and industrial journal. Bull Mech Eng 9:19-25

Adonin AN (1973) To the calculation of the loads acting on the bars of a deep pump. Azerbaijan Oil Industry 4:44-46

Ahmedov BB, Kerimov SK, Jafarov EA (2015) The study of the kinematic parameters of a beamless rocking machine. J Mach Sci 4(1):29-32

Ahmedov BB, Najafov AM, Abdullaev AI (2018) Determination of the kinematic parameters of the new constructive solution of the beamless sucker-rod pump. J Struct Eng Appl Mech 1(3):128-135

Aliverdizade KS (1951) Balanced individual drives of a deep-well pumping unit (pumping units). Azneftizdat, Baku

Chicherov LG, Molchanov GV, Rabinovich AM (1987) Calculation and design of oilfield equipment. Nedra, Moscow

Gimatudinov SK (1983) Reference guide for the design and development of oil fields. Nedra, Moscow

Ivanovsky VN, Darishchev VI, Kashtanov VS, Sabirov AA, Peking SS (2002) Well pumping installations for oil production: oil and gas, Moscow

Mishchenko IT (2003) Downhole oil production: Textbook for universities. Federal State Unitary Enterprise Publishing House, The Gubkin Russian State University of Oil and Gas, Moscow

Virnovsky AS (1971) Theory and Practicum of Deep-Oil Production. Nedra, Moscow

Publisher's Note Springer Nature remains neutral with regard to jurisdictional claims in published maps and institutional affiliations. 